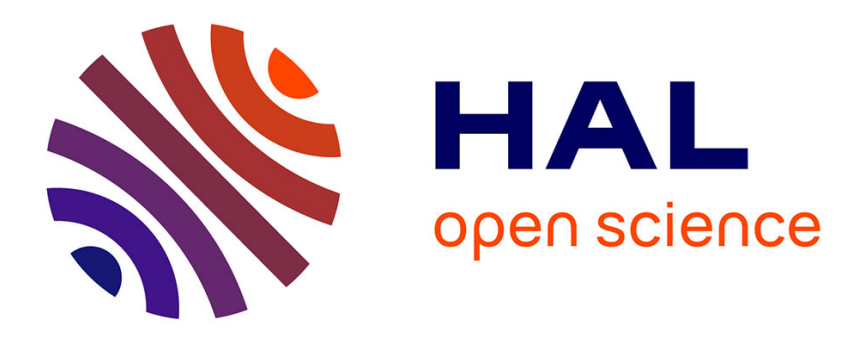

\title{
Limite de résolution de quelques méthodes de mesure de la densité de grains
}

\author{
Mohamed Dellagi
}

\section{To cite this version:}

Mohamed Dellagi. Limite de résolution de quelques méthodes de mesure de la densité de grains. Revue de Physique Appliquée, 1966, 1 (4), pp.297-299. 10.1051/rphysap:0196600104029701 . jpa-00242751

\section{HAL Id: jpa-00242751 https://hal.science/jpa-00242751}

Submitted on 1 Jan 1966

HAL is a multi-disciplinary open access archive for the deposit and dissemination of scientific research documents, whether they are published or not. The documents may come from teaching and research institutions in France or abroad, or from public or private research centers.
L'archive ouverte pluridisciplinaire HAL, est destinée au dépôt et à la diffusion de documents scientifiques de niveau recherche, publiés ou non, émanant des établissements d'enseignement et de recherche français ou étrangers, des laboratoires publics ou privés. 


\title{
LIMITE DE RÉSOLUTION DE QUELQUES MÉTHODES DE MESURE DE LA DENSITÉ DE GRAINS
}

\author{
Par Mohamed Dellagi, \\ Laboratoire de Physique, Faculté des Sciences, Tunis.
}

\begin{abstract}
Résumé. - Les calculs sont faits dans un modèle où les grains d'argent sont égaux et sphériques (diamètre $\alpha$ ) et où les projections de leur centre sur la direction générale de la trace sont distribuées au hasard, avec une densité moyenne g. A comptage constant et pour $\alpha g>0,96$ la méthode de la longueur moyenne des paquets sépare deux espèces de traces, caractérisées par des $g$ différents, mieux que la méthode de la longueur moyenne des lacunes. A parcours constant, dans le cas du comptage du nombre de lacunes ou de la mesure de leur longueur intégrée, on applique les résultats à l'estimation du parcours mininum irréductible permettant la séparation.
\end{abstract}

Abstract. - Calculations are made, using a model with equivalent and spherical grains, and where the projection of their centers is distribued at random, with a mean density g. At constant counting, and for $g=0.6$, the mean-length bundle method separates two kinds of tracks, defined by different $g$, and seems better than the method using the mean length of the gaps.

At constant range, in the case where the number of gaps is counted, or their integrated length measured, the results are applied to the estimation of the minimum irreductible path length allowing the separation.

Soit, dans une émulsion nucléaire, un échantillon de traces $e$, formé d'un mélange de deux souséchantillons $e_{1}$ et $e_{2}$ issus des populations $p_{1}$ et $p_{2}$ caractérisées, respectivement, par les densités de grains $g$ et $g+\Delta g$. On se propose de calculer le plus petit intervalle $\Delta g$ que les différentes méthodes de mesure de $g$ peuvent mettre en évidence, quand on se place dans les conditions théoriques décrites par l'introduction de la précédente note [1].

Pour fixer les idées, on suppose que les traces sont horizontales, que $e_{1}$ et $e_{2}$ se présentent en proportions à peu près égales et que la fluctuation d'un caractère est d'origine purement statistique. On désigne par $\langle a\rangle$ et $\sigma_{a}^{2}$ l'espérance mathématique et la variance d'une grandeur aléatoire $a$.

Les méthodes couramment utilisées se pratiquent à comptage, ou à parcours constant.

\section{Mesures à comptage $n$ constant. - 1) MÉTH ODE} de La Longueur moyenne des Lacunes. Chaque trace est caractérisée par $\bar{l}$ longueur moyenne de $n$ lacunes. Soient $d, d_{1}, d_{2}$ les distributions de $l$ relatives à $e, e_{1}, e_{2} ;$ la donnée immédiate est $d$ superposition de $d_{1}$ et $d_{2}$; pour les $\bar{l}$ de $d_{1}$, on a : $\langle\vec{l}\rangle=1 / \mathrm{g}$ et $\sigma_{\bar{l}}^{2}=1 / \mathrm{ng}^{2}$. Le nombre $n$ est pris assez grand pour que $d_{1}$ et $d_{2}$ soient normales (théorème de la limite centrale). On admet que $e_{1}$ et $e_{2}$ sont séparés quand $d$ présente une dépression au sommet : on pose que cela a lieu lorsque $d_{2}$ est distant de $d_{1}$ d'au moins la largeur à mi-hauteur, $2,35 \sigma$, de $d_{1}$. La limite de résolution, $\Delta g$, de la méthode est donc donnée par :

$$
|\Delta<\bar{l}>| \Delta g \mid \Delta g=2,35 . \sigma_{\bar{l}} .
$$


Ce raisonnement est typique, et dans la suite, on l'utilisera sans le reproduire.

On trouve donc :

$$
\Delta g=2,35 \cdot g / \sqrt{n} .
$$

Le diamètre $\alpha$ des grains d'argent n'apparaît pas dans ce résultat ; $\alpha$ peut varier d'une trace à l'autre, sans que $d$ en soit affectée [2]. Par contre, dans la suite, on suppose $\alpha$ constant dans tout l'échantillon $e$.

2) Méthode de la longueur moyenne des PAQUETS. - On caractérise chaque trace $\mathrm{pa}^{\mathrm{r}} \bar{b}$ longueur moyenne de $n$ paquets. On a [3] successi-

$\frac{\alpha \mathrm{g}}{\mathrm{I}} \quad \frac{0,2}{2,44} \quad \frac{0,4}{1,70} \quad \frac{0,6}{1,36} \quad \frac{0,8}{1,14} \quad \frac{1,0}{0,975} \quad \frac{1,2}{0,856}$

La limite de résolution est $I$ fois celle de la méthode précédente; on doit préférer cette dernière quand $\alpha g<0,96$, car alors $I>1$.

II. Mesures à parcours $\boldsymbol{R}$ constant. - Une portion de trace de longueur $R$ est caractérisée par le nombre $N$ de lacunes qu'elle contient, ou par leur longueur intégrée $L$. Quand $\langle N\rangle$ est suffisamment élevée, $N$ et $L$ ont des distributions normales, dont les éléments peuvent se déduire des calculs de Domb [4].

1) Comptage de $N$. - On sait (formules 34 et 36 de [4]) que :

$$
\begin{gathered}
\langle N>=(1+R g) \exp (-g \alpha) \\
\sigma_{N}^{2}=g R \exp (-g \alpha)[1-2 g \alpha \exp (-g \alpha)] \\
+\exp (-g \alpha)\left[1-\exp (-g \alpha)\left(1+2 g \alpha-g^{2} \alpha^{2}\right)\right] .
\end{gathered}
$$

Supposant $R \gg \alpha$ et $R g \gg 1$, on obtient :

$$
\Delta g=2,35 \sqrt{J / R}
$$

avec

$$
J=[\exp (g \alpha)-2 \alpha g] / g[\alpha-(1 / g)]^{2} .
$$

Le graphique montre les courbes représentatives de $J$ en fonction de $\alpha$ pour $g=1 \mu^{-1}$ et $g=4 \mu^{-1}$; asymptotes d'équation $\alpha=1 / g$. On constate, en dehors du minimum banal d'abscisse $\alpha=0$ et d'ordonnée $J=g$, l'existence d'un autre minimum de coordonnées $\alpha_{\mathrm{m}}, J_{\mathrm{m}}$ dont le lieu, quand $g$ varie, est un arc d'hyperbole équilatère d'équation $J_{\mathrm{m}}=7,58 / \alpha_{\mathrm{m}}$ avec :

$$
g \alpha_{m}=2,38 .
$$

vement avec des notations analogues aux précédentes :

$$
\begin{aligned}
<\bar{b}> & =[\exp (\alpha g)-1] / g \\
\sigma_{\bar{b}}^{2} & =[1-\exp (-2 g \alpha)-2 \alpha g \\
& \exp (-\alpha g)] / n g^{2} \exp (-2 g \alpha) \\
\Delta g & =2,35 g . I / \sqrt{n}
\end{aligned}
$$

où

$I=[\exp (2 \alpha g)-1-2 \alpha g$

$\exp (g \alpha)]^{1 / 2} /[1-\exp (\alpha g)+\alpha g \exp (g \alpha)]$

$I$ est une fonction décroissante de $\alpha g$; le tableau ci-dessous en donne quelques valeurs pour $\alpha g$; variant de 0,2 à 2,4 :

$\begin{array}{cccccc}1,4 & \frac{1,6}{1,8} & \frac{2,0}{2,2} & \frac{2,4}{\overline{7}} \\ 0, \overline{770} & 0, \overline{698} & 0, \overline{638} & 0, \overline{585} & 0,540 & 0,500\end{array}$

2) Mesure de $L$. - On a rigoureusement (formules 64 et 65 de [4]) :

$$
<L>=R \exp (-g \alpha)
$$

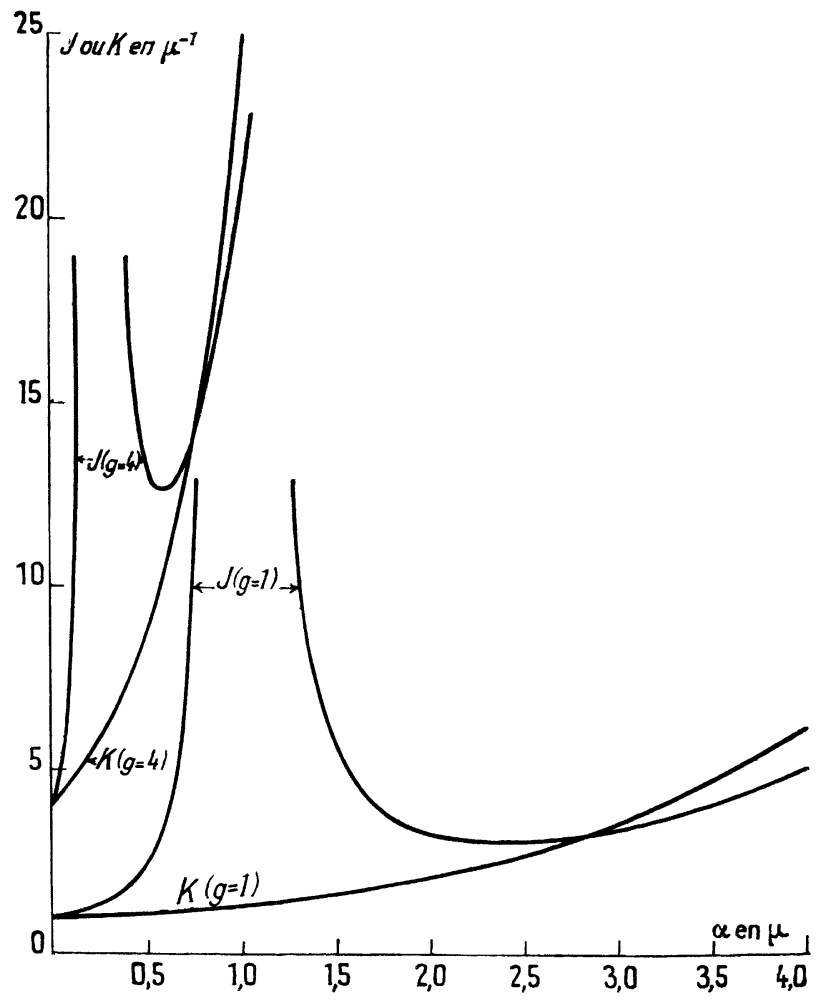

Limite de résolution pour un parcours $R$ donné, de la méthode : 1) du comptage de lacunes

$$
\Delta g=2,35 \sqrt{J / R}
$$

2) de la longueur intégrée de lacunes

$$
\Delta g=2,35 \sqrt{K / R} \text {. }
$$


et pour $R>\alpha$ :

$$
\begin{array}{r}
\sigma_{\mathrm{L}}^{2}=2 \exp (-g \alpha)[1-\exp (-g \alpha)](R g-1) / g^{2} \\
+\exp (-2 g \alpha)\left[\alpha^{2}+(2 \alpha / g)-2 R \alpha\right] .
\end{array}
$$

D'où :

$$
\Delta g=2,35 \sqrt{(K / R)[1-(\varepsilon / R)]}
$$

en posant :

$$
K=2[\exp (g \alpha)-1-g \alpha] / g \alpha^{2}
$$

et

$$
\varepsilon=(1 / g)-(1 / K) .
$$

On supposera $R g \gg 1$; cela donne alors :

$$
\Delta g=2,35 \sqrt{K / R},
$$

Les courbes représentatives de $K$ en fonction de $\alpha$, pour $g=1 \mu^{-1}$ et $g=4 \mu^{-1}$ sont dessinées sur le graphique.

Quand $\alpha, g, \Delta g$ sont donnés, aux deux méthodes précédentes correspondent respectivement les parcours minima irréductibles

$$
(2,35 / \Delta g)^{2} J \text { et }(2,35 / \Delta g)^{2} K .
$$

Les courbes $J$ et $K$, relatives à la même valeur de $g$, se coupent en deux points qui, $g$ variant, se déplacent l'un sur la droite $\alpha=0$ et l'autre sur l'hyperbole équilatère d'équation $J_{\mathrm{c}}=10,0 / \alpha_{\mathrm{c}}$, avec $g \alpha_{c}=2,92$. On vérifie sur le graphique que pour $\alpha g<2,92$ et c'est le cas le plus courant, $K$ est inférieur à $J$ : ainsi est justifiée, par le calcul, la supériorité de la méthode de la longueur intégrée des lacunes sur la méthode du comptage.

Des nécessités d'ordre technique peuvent, malgré tout, imposer cette dernière. Si on est maître du choix de $\alpha$, on le prendra petit, ou voisin de $\alpha_{\mathrm{m}}$ et de toute façon différent de $1 / \mathrm{g}$. Dans le cas contraire, et si $\alpha$ est voisin de $1 / g$, il est recommandé de le gonfler artificiellement d'un incrément $r$ tel que $\alpha_{n}=\alpha+r$, ce qui revient à ne compter que les lacunes de longueur supérieure à $r$; le minimum irréductible vaut alors $(2,35 / \Delta g)^{2} J_{\mathrm{m}}$. Exemple : Pour $g=4 \mu^{-1}$, on d'après a (1) : $\alpha_{\mathrm{m}}=0,6 \mu$; si on sait que $\alpha$ est voisin de $0,4 \mu$ on comptera les lacunes plus longues que $0,2 \mu$; un parcours valant $\left[(2,35 / \Delta g)^{2} .12,7\right] \mu$, suffira pour séparer $e_{1}$ de $e_{2}$. Le comptage de toutes les lacunes, outre les difficultés introduites par celles qui sont voisines de zéro [5], aurait nécessité un parcours $19,5 / 12,7=1,53$ fois plus long.

Manuscrit reçu le 19 juillet 1966.

\section{BIBLIOGRAPHIE}

[1] Dellagi, C.R. Acad. Sc., 1966, 262, 1092.

[2] Dellagi, C.R. Acad. Sc., 1965, 261, 2318.

[3] Barkas, Nuclear Research Emulsions, Academic Press, New York, 1963, p. 395.
[4] Dомв, Proc. Cambridge Phil. Soc., 1947, 43, 329.

[5] Cortini-Luzzato-Tomasini-Manfredini, Nuovo Cimento, 1958, 9, nº 4, 707. 